\title{
Alterações Lipídicas em Pacientes com Câncer Colorretal em Fase Pós-Operatória: Ensaio Clínico Randomizado e Duplo-Cego com Fungos Agaricus sylvaticus
}

\author{
Lipidic Alterations in Patients with Colorectal Cancer in Post-Surgery Phase: A \\ Randomized and Double-Blind Clinical Trial with Agaricus sylvaticus Fungus
}

\author{
RENATACOSTAFORTES ${ }^{1}$, ANDRESALIMAMELO ${ }^{2}$ VIVIANE LACORTE RECÔVA $^{2}$; \\ MARIARITACARVALHO GARBI NOVAES ${ }^{3}$
}

${ }^{1}$ Universidade de Brasília (DF)/ Faculdade de Ciências e Educação Sena Aires (GO); ${ }^{2}$ Faculdade de Medicina da Escola Superior em Ciências da Saúde do Distrito Federal/ ESCS/ FEPECS (DF); ${ }^{3}$ Universidade de Brasília (DF)/ Faculdade de Medicina da Escola Superior em Ciências da Saúde do Distrito Federal ESCS/ FEPECS (DF) - Brasil.

FORTES RC; MELO AL; RECÔVA VL; NOVAES MRCG. Alterações Lipídicas em Pacientes com Câncer Colorretal em Fase PósOperatória: Ensaio Clínico Randomizado e Duplo-Cego com Fungos Agaricus sylvaticus. Rev bras Coloproct, 2008;28(3): 281-288.

RESUMO: Introdução: Alterações no metabolismo lipídico são comuns em pacientes com câncer. Fungos medicinais podem exibir atividade hipolipidêmica. Objetivo: Avaliar os efeitos da suplementação dietética com fungos Agaricus sylvaticus no perfil lipídico de pacientes com câncer colorretal em fase pós-operatória. Métodos: Ensaio clínico randomizado, duplo-cego, placebocontrolado, realizado no Hospital de Base do Distrito Federal por seis meses. Amostra constituída por 56 pacientes, estádios I, II e III, separados em dois grupos: placebo e suplementado com Agaricus sylvaticus $(30 \mathrm{mg} / \mathrm{kg} / \mathrm{dia})$. Resultados analisados pelos programas Microsoft Excel 2003 e SPSS $14.0 \mathrm{com} p=0.05$. Resultados: $O$ grupo Agaricus sylvaticus apresentou níveis séricos iniciais de colesterol total de $\mathbf{2 0 7 . 3 6} \pm \mathbf{5 2 . 6 7} \mathrm{mg} / \mathrm{dL}$, lipoproteína de baixa densidade de $120.79 \pm 44.02 \mathrm{mg} / \mathrm{dL}$ e triglicérides de $181.64 \pm 187.52 \mathrm{mg} / \mathrm{dL}$. Após seis meses de suplementação, observou-se redução para $191.11 \pm 39.72 \mathrm{mg} / \mathrm{dL}(p=0.01), 103.08 \pm 39.20 \mathrm{mg} /$ dL $(p=0.0001)$ e $168.04 \pm 146.91 \mathrm{mg} / \mathrm{dL}(p=0.18)$, respectivamente. No grupo placebo, observou-se aumento não-significativo de colesterol total $(p=0.08)$ e aumento significativo de lipoproteína de baixa densidade $(p=0.01)$ e triglicérides $(p=0.0001)$. Não foram observadas, em ambos os grupos, alterações significantes nos níveis de lipoproteína de alta densidade e lipoproteína de muito baixa densidade. Conclusão: Os resultados sugerem que a suplementação dietética com Agaricus sylvaticus pode melhorar significativamente o perfil lipídico de pacientes com câncer colorretal em fase pós-operatória.

Descritores: Agaricus sylvaticus, fungos medicinais, alterações metabólicas, metabolismo lipídico, câncer colorretal.

\section{INTRODUÇÃO}

O câncer, enfermidade crônica multicausal, caracterizado pelo crescimento e desenvolvimento desordenado das células, é uma das maiores causas de óbito da humanidade, sendo considerado um sério problema de saúde pública ${ }^{1}$.
O desenvolvimento das neoplasias malignas resulta da interação entre fatores endógenos e ambientais, destacando-se a dieta como um dos principais fatores de risco na etiologia dos diversos tipos de câncer ${ }^{1-3}$.

Dentre as principais alterações metabólicas induzidas pelos tumores avançados, observam-se

\footnotetext{
Trabalho realizado no Hospital de Base da Secretaria de Estado de Saúde do Distrito Federal, Brasília, DF. Endereço: SMHS - Área Especial Quadra 101. CEP: 70.330-150 - Brasília - DF - Brasil.

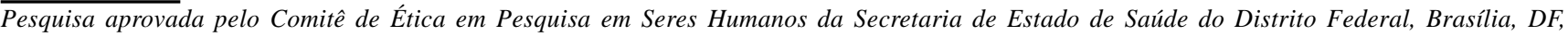
em 08 de julho de 2004.

Não há conflitos de interesse que possam influenciar elou ter influenciado os resultados da pesquisa elou o conteúdo do trabalho.
} 
depleção dos depósitos lipídicos, aumento da lipólise, aumento do turnover de glicerol e ácidos graxos livres, redução da lipogênese e, conseqüente, hiperlipidemia ${ }^{2}$.

Evidências científicas têm demonstrado que a suplementação dietética com fungos medicinais é capaz de exibir atividade hipolipidêmica em estudos experimentais ${ }^{4-6}$ e clínicos ${ }^{7}$ por conterem substâncias que atuam na regulação do metabolismo lipídico ${ }^{3}$.

O objetivo deste estudo foi avaliar os efeitos da suplementação dietética com fungos Agaricus sylvaticus no perfil lipídico de pacientes com câncer colorretal em fase pós-operatória atendidos no Ambulatório de Proctologia do Hospital de Base do Distrito Federal, Brasil.

\section{MATERIAIS E MÉTODOS}

\section{Metodologia do estudo}

O estudo constitui-se de um ensaio clínico randomizado, duplo-cego, placebo-controlado, com alocação aleatória dos pacientes. Foi aprovado pelo Comitê de Ética em Pesquisa da Secretaria de Estado de Saúde do Distrito Federal, sob o protocolo ${ }^{\circ}$ 051/ 2004. O termo de consentimento livre e esclarecido foi obtido dos pacientes, cuja participação foi voluntária. O trabalho foi desenvolvido no Ambulatório de Proctologia do Hospital de Base do Distrito Federal Brasil no período de novembro de 2004 a julho de 2006.

\section{Casuística}

A amostra foi constituída por 56 pacientes ( 24 homens e 32 mulheres) com câncer colorretal, estádios I ( $n=12)$, II $(n=16)$ e III $(n=28)$, separados em dois grupos: placebo e suplementado com fungo Agaricus sylvaticus.

Os pacientes foram selecionados de acordo com os seguintes critérios de inclusão: pacientes com diagnóstico confirmado de câncer colorretal, de três meses a dois anos de intervenção cirúrgica, idade superior a vinte anos; e de exclusão: gestantes, lactantes, acamados, deficientes físicos, pacientes em uso de terapia alternativa, portadores de outras doenças crônicas não-transmissíveis e em processo de metástase.

\section{Extrato do fungo Agaricus sylvaticus}

O fungo Agaricus sylvaticus, foi obtido de um produtor, credenciado pela Empresa Brasileira de Agropecuária - Embrapa, da região de Tapiraí, Estado de São Paulo. O extrato do fungo foi obtido por imersão do material desidratado em água quente por 30 minutos, liquidificado, peneirado e seco em dissecador. A análise da composição do Agaricus sylvaticus foi realizada pelo Japan Food Research Laboratories Center e revelou a presença de carboidratos $(18,51 \mathrm{~g} /$ $100 \mathrm{~g})$, lipídeos $(0,04 \mathrm{~g} / 100 \mathrm{~g})$, ergosterol $(624 \mathrm{mg} / 100 \mathrm{~g})$, proteínas $(4,99 \mathrm{~g} / 100 \mathrm{~g})$, aminoácidos (arginina- $1,14 \%$; lisina-1,23\%; histidina- $0,51 \%$, fenilalanina- $0,92 \%$, tirosina- $0,67 \%$, leucina- $1,43 \%$, metionina- $0,32 \%$, valina$1,03 \%$, alanina- $1,28 \%$, glicina- $0,94 \%$, prolina- $0,95 \%$, ácido glutâmico-3,93\%, serina- $0,96 \%$, treonina- $0,96 \%$, ácido aspártico- $1,81 \%$, triptofano- $0,32 \%$, cisteína$0,25 \%)$ e micronutrientes em quantidades-traço.

$\mathrm{O}$ extrato seco foi transformado em comprimidos, seguindo procedimento farmacotécnico. A dosagem do fungo administrada aos pacientes foi equivalente a $30 \mathrm{mg} / \mathrm{kg} / \mathrm{dia}$ (considerando o peso médio da população estudada), fracionada em duas tomadas diárias (seis comprimidos por dia, sendo três comprimidos pela manhã e três à tarde, nos intervalos entre as refeições) durante um período de seis meses. Ao grupo de pacientes que recebeu o placebo, foram administrados os comprimidos nas mesmas quantidades, com os mesmos excipientes e valor calórico, porém sem o extrato do fungo Agaricus sylvaticus.

\section{Evolução clínica}

Os pacientes foram acompanhados por um período de seis meses, sendo que nos três primeiros meses, foram realizadas consultas quinzenais para a avaliação clínica e, nos últimos três meses, as consultas passaram a ser a cada 30 dias.

Todos os pacientes permaneceram com dieta habitual, embora durante o tratamento tenham recebido orientações gerais sobre como manter uma alimentação saudável. Após seis meses de acompanhamento foi indicada dieta individualizada para todos os pacientes e os mesmos foram encaminhados a outros profissionais da área de saúde, quando necessário.

Foram realizados três exames laboratoriais para análise do perfil lipídico dos pacientes: antes do início da suplementação, após três meses de tratamento e ao final do tratamento.

Todos os pacientes foram contactados pelos pesquisadores, semanalmente, via telefone, para esclarecimentos de dúvidas, verificação do uso adequado do Agaricus sylvaticus e confirmação do agendamento, garantindo maior adesão ao tratamen- 
to e controle sobre a continuidade do paciente no estudo.

Foram considerados desistentes os pacientes que compareceram somente às primeiras consultas, que não vieram às consultas durante o período de seis meses, que fizeram menos de três exames. Aqueles que faleceram antes do final do tratamento não foram incluídos na amostragem.

\section{Avaliação laboratorial}

A coleta do sangue foi realizada seguindo o critério de $12 \mathrm{~h}$ de jejum dos pacientes. O material coletado foi depositado em tubos secos à vácuo para a obtenção do soro, seguindo os protocolos recomendados pela Sociedade Brasileira de Patologia Clínica para a Coleta de Sangue Venoso ${ }^{8}$. Os exames foram realizados no Laboratório de Patologia Clínica do Hospital de Base da Secretaria de Estado de Saúde do Distrito Federal. As amostras coletadas foram centrifugadas e analisadas em aparelho TARGA 3000 - Random Access Chemistry Analiser, seguindo os procedimentos do laboratório. Para a determinação da análise de colesterol total (CT), lipoproteína de alta densidade (HDL colesterol/HDL-c), triglicerídeos (TG), utilizouse o reagente para análise automatizada col-HDL monofase marca Wiener. Os valores de lipoproteína de baixa densidade (LDL colesterol/ LDL-c) e lipoproteína de muito baixa densidade (VLDL colesterol/ VLDL-c) foram calculados pela fórmula de Friedewald (válida para triglicerídeo $<400 \mathrm{mg} / \mathrm{dL}$ ): LDL $=$ colesterol total $-\mathrm{HDL}-($ triglicerídeos/5). Os resultados dos exames foram analisados de acordo com os valores de referência padronizados pelo Laboratório de Análises Clínicas do referido hospital.

\section{Análise estatística}

Os valores dos lipídeos plasmáticos apresentados foram comparados e analisados por meio dos testes estatísticos T-student e F, utilizando-se os programas Microsoft Excel 2003 e SPSS (Statistical Package of the Social Sciences, SPSS Inc, Chicago, EUA) para Windows versão 14.0, com valor de significância de $p \leq 0.05$.

\section{RESULTADOS}

Após seis meses de acompanhamento no Ambulatório de Proctologia do Hospital de Base do Distrito Federal, 56 pacientes com câncer colorretal conclu- íram o estudo, sendo 32 mulheres $(57,1 \%)$ e 24 homens $(42,9 \%)$, separados nos grupos que receberam o placebo e o fungo Agaricus sylvaticus.

Os pacientes do grupo placebo $(n=28)$ tinham idade média de 59,14 $\pm 12,95$ anos. Com relação ao gênero e ao estadiamento, $57,1 \%(n=16)$ eram do sexo feminino, sendo três do estádio I, sete do II e seis do III, e $42,9 \%(n=12)$ do sexo masculino, um do estádio I, três do II e oito do III.

Os pacientes do grupo que recebeu o Agaricus sylvaticus $(n=28)$ tinham idade média de 56,34 $\pm 15,53$ anos. Quanto ao gênero, $57,1 \%(n=16)$ eram do sexo feminino, sendo seis do estádio I, dois do II e oito do III, e 42,9\% ( $n=12)$ do sexo masculino, dois do estádio I, quatro do II e sete do estádio III.

Em relação à análise de colesterol total, foram observados os seguintes resultados: o grupo placebo tinha um colesterol total inicial de $192.71 \pm 35.70 \mathrm{mg} / \mathrm{dL}$, após três meses foi observado um aumento (de $192.71 \pm 35.70 \mathrm{mg} / \mathrm{dL}$ para $204.68 \pm 56.09 \mathrm{mg} / \mathrm{dL}, p=$ $0.08)$ e, no sexto mês, houve novamente um aumento (de $192.71 \pm 35.70 \mathrm{mg} / \mathrm{dL}$ para $203.32 \pm 48.78 \mathrm{mg} / \mathrm{dL}, p=$ 0.08 ), porém estas alterações não foram estatisticamente significativas, figura 1 .

O grupo suplementado com Agaricus sylvaticus tinha um colesterol total inicial de $207.36 \pm 52.67 \mathrm{mg} / \mathrm{dL}$, com três meses de $194.68 \pm 44.66 \mathrm{mg} / \mathrm{dL}$, e após seis meses de $191.11 \pm 39.72 \mathrm{mg} / \mathrm{dL}$ com redução significativa dos níveis plasmáticos de colesterol total $\operatorname{com} p=$ 0.05 e $p=0.01$, respectivamente, figura 1 .

$\mathrm{O}$ valor inicial do HDL-c do grupo placebo foi de $52.40 \pm 14.91 \mathrm{mg} / \mathrm{dL}$, após três meses $61.29 \pm 39.42 \mathrm{mg} /$ $\mathrm{dL}(p=0.13)$ e seis meses $55.89 \pm 28.61 \mathrm{mg} / \mathrm{dL}(p=0.28)$, demonstrando aumento não-significativo, quando comparado com os resultados iniciais, figura 2 .

O HDL-c inicial do grupo suplementado com Agaricus sylvaticus foi de $51.18 \pm 19.06 \mathrm{mg} / \mathrm{dL}$, apresentando aos três meses redução não-significativa para $50.75 \pm 12.00 \mathrm{mg} / \mathrm{dL}, p=0.41$, e após seis meses aumento para $52.04 \pm 13.07 \mathrm{mg} / \mathrm{dL}, p=0.36$, figura 3 .

Os valores iniciais do LDL-c do grupo placebo foram de $109.67 \pm 32.53 \mathrm{mg} / \mathrm{dL}$ com aumento significativo após três meses $124.71 \pm 38.95 \mathrm{mg} / \mathrm{dL}(p=0.0001)$ e seis meses $121.07 \pm 41.80 \mathrm{mg} / \mathrm{dL}(p=0.01)$, quando comparado com os resultados iniciais, figura 2.

O grupo que recebeu Agaricus sylvaticus apresentou inicialmente valores de LDL-c de $120.79 \pm 44.02 \mathrm{mg} / \mathrm{dL}$, observou-se redução significativa com três meses para $107.33 \pm 43.26 \mathrm{mg} / \mathrm{dL}, p=0.01$ 


\section{Resultados dos níveis plasmáticos de colesterol total}

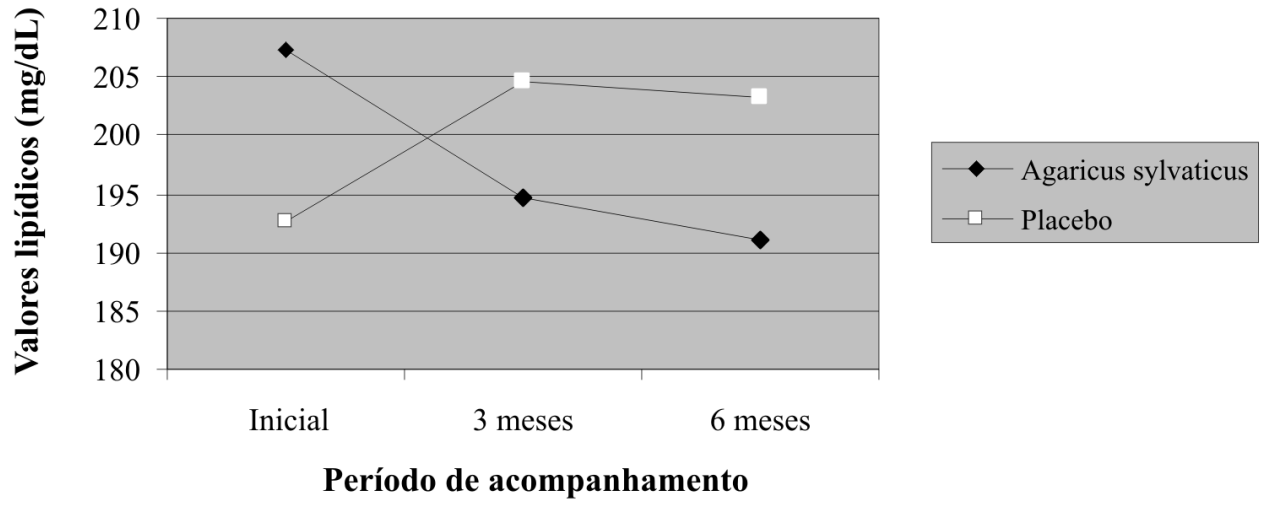

Figura 1 - Níveis plasmáticos de colesterol total de pacientes com câncer colorretal após seis meses de segmento em ensaio clínico randomizado.

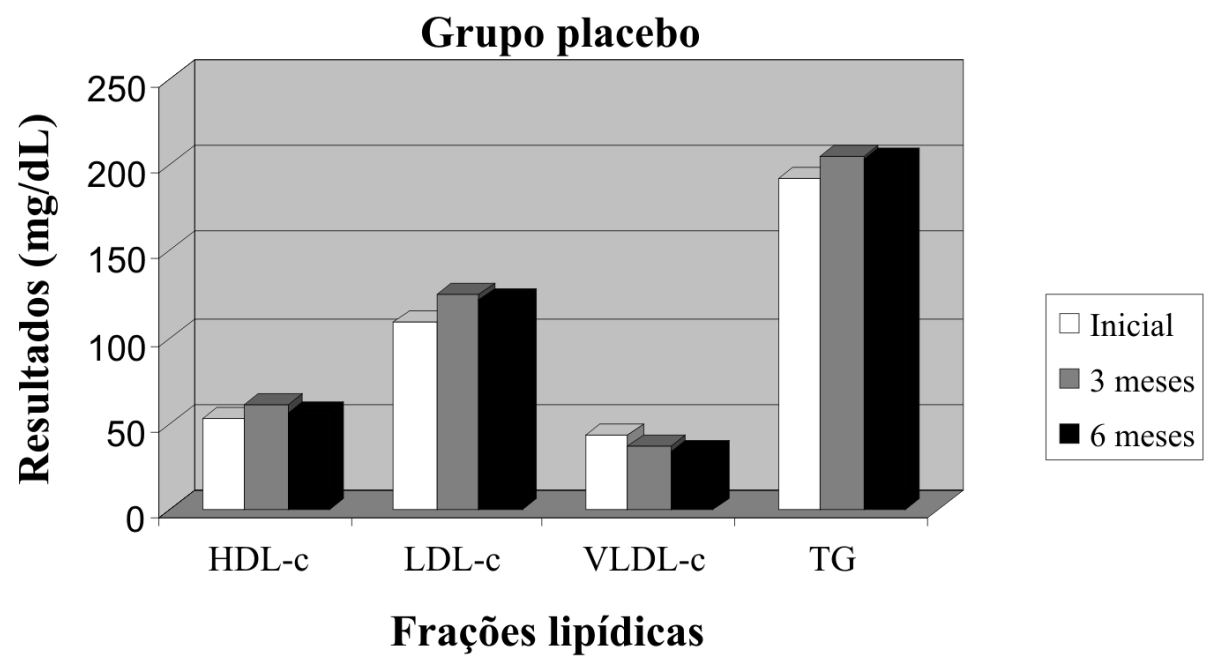

Figura 2 - Níveis plasmáticos de HDL-c, LDL-c, VLDL-c e TG dos pacientes do grupo placebo durante o período de acompanhamento.

e com seis meses para $103.80 \pm 39.20 \mathrm{mg} / \mathrm{dL}, p=0.0001$, figura 3 .

O grupo placebo tinha uma taxa de VLDL-c inicial de $42.75 \pm 49.61 \mathrm{mg} / \mathrm{dL}$, com diminuição para $36.36 \pm 31.87 \mathrm{mg} / \mathrm{dL}, p=0.10$ aos três meses e após seis meses, nova diminuição para $33.21 \pm 14.44 \mathrm{mg} / \mathrm{dL}$, $p=0.15$, porém todas essas alterações não foram estatisticamente significativas, figura 2.

O grupo Agaricus sylvaticus apresentou redução não-significativa de VLDL-c com três meses de suplementação (de $36.79 \pm 35.62 \mathrm{mg} / \mathrm{dL}$ para $36.46 \pm 34.79 \mathrm{mg} / \mathrm{dL}, p=0.46$ ), e aumento não-signifi- cativo com seis meses (de $36.79 \pm 35.62 \mathrm{mg} / \mathrm{dL}$ para $39.17 \pm 39.72 \mathrm{mg} / \mathrm{dL}, p=0.31)$, figura 3 .

No grupo placebo, observou-se aumento significativo dos níveis séricos de TG com três meses (de $137.93 \pm 59.19 \mathrm{mg} / \mathrm{dL}$ para $157.54 \pm 68.45 \mathrm{mg} / \mathrm{dL}, p=0.01) \mathrm{e}$ após seis meses (de $137.93 \pm 59.19 \mathrm{mg} / \mathrm{dL}$ para $169.65 \pm 89.13 \mathrm{mg} / \mathrm{dL}, p=0.0001$ ), figura 2. Esses achados não foram encontrados no grupo Agaricus sylvaticus que apresentou redução das taxas de TG com três meses (de $181.64 \pm 187.62 \mathrm{mg} / \mathrm{dL}$ para $174.79 \pm 168.17 \mathrm{mg} / \mathrm{dL}, p=0.33$ ), e após seis meses (de $181.64 \pm 187.62 \mathrm{mg} / \mathrm{dL}$ para $168.04 \pm 146.91 \mathrm{mg} / \mathrm{dL}, p=0.18$ ) de suplementação, figura 3 . 


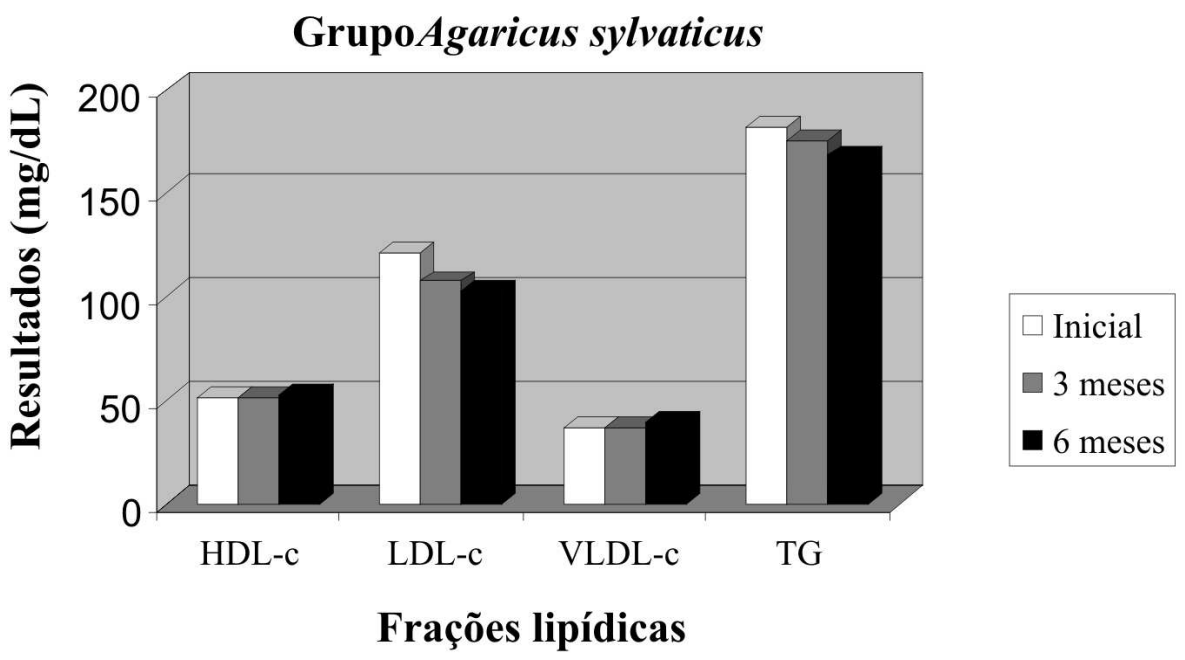

Figura 3 - Níveis plasmáticos de HDL-c, LDL-c, VLDL-c e TG do grupo Agaricus sylvaticus durante o período de suplementação.

\section{DISCUSSÃO}

Foram encontrados neste estudo, 57,1\% de pacientes do sexo feminino e, $42,9 \%$ do sexo masculino. Esses dados corroboram com os estudos de Pinho et $a l^{9}$ que apontam predominância de câncer colorretal no sexo feminino comparado com o masculino na regional estudada. Porém, na literatura, há controvérsias em relação à incidência deste tipo de neoplasia onde alguns estudos apontam que o mesmo incide prevalentemente em homens ${ }^{10}$, e outros justificam igual distribuição em ambos os sexos ${ }^{11}$.

A idade média dos pacientes foi de $59,14 \pm 12,95$ anos e 56,34 $\pm 15,53$ anos nos grupos placebo e Agaricus sylvaticus, respectivamente. A idade mínima foi de 23 anos e a máxima de 79 anos. Tais resultados são confirmados pela literatura que indica maior incidência de câncer colorretal na faixa etária entre 50 e 70 anos com possibilidades aumentadas de desenvolvimento a partir da quarta década de vida ${ }^{12}$.

Em relação ao estadiamento, 50,00\% dos pacientes dos grupos placebo e Agaricus sylvaticus pertenciam ao estádio III, tanto pelo sistema TNM como pela classificação de Dukes. Resultados similares foram encontrados no estudo de Saad-Hossne et al ${ }^{10}$, demonstrando retardo do diagnóstico na maioria dos pacientes o que pode comprometer significativamente o prognóstico.

Evidências científicas têm demonstrado que, dentre as principais alterações metabólicas induzidas pelas neoplasias malignas, destacam-se a depleção dos depósitos lipídicos, o incremento da lipólise e do turnover de glicerol e ácidos graxos livres, além da redução da lipogênese e, conseqüente, hiperlipidemia ${ }^{2,13}$. Esta, por sua vez, pode estar relacionada à inibição da atividade da enzima lipase lipoprotéica plasmática. Tanto a diminuição na atividade da lipase lipoprotéica quanto o aumento na lipogênese hepática são mediados por fator de necrose tumoral alfa $(\mathrm{TNF}-\alpha)$ e interleucina 1 $(\mathrm{IL}-1)^{14}$.

Além da redução da atividade da lipase lipoprotéica, as alterações no metabolismo lipídico estão relacionadas ao aumento da lipase hormônio-sensível. Esses fatores culminam com consequiente aumento na oxidação lipídica, resultando no incremento de ácidos graxos livres, glicerol e hipertrigliceridemia secundária ao decréscimo na depuração sérica dos triglicerídeos VLDL pela diminuição da atividade da lipase lipoprotéica. Acredita-se que essas alterações metabólicas estejam sob o controle dos mediadores do câncer como o TNF- $\alpha$. Porém, ainda não está estabelecido se essas citocinas têm ação direta sob o consumo das reservas lipídicas nos pacientes com neoplasias malignas. Evidenciou-se, recentemente, a existência de um fator de mobilização de lipídeos sintetizado pelo tumor ou hospedeiro com câncer ${ }^{15}$.

Os principais mecanismos envolvidos com o aumento na taxa de lipólise nos pacientes com câncer incluem redução da ingestão oral, perda ponderal, resposta de estresse à doença, resistência à insulina e liberação de fatores lipolíticos pelo tumor ${ }^{13,14}$. 
No presente estudo, observou-se, no grupo Agaricus sylvaticus, redução significativa dos níveis plasmáticos de CT e LDL-c durante todo o período de suplementação. Resultados inversos foram observados no grupo placebo, sugerindo a presença de substâncias bioativas nos fungos Agaricus sylvaticus capazes de reduzir essas frações lipídicas.

Acredita-se que os fungos medicinais são capazes de modular o processo de carcinogênese nos diversos estádios da doença por meio de distintos mecanismos. Porém, os mecanismos de ação dos princípios ativos presentes nos fungos Agaricales e em outros fungos medicinais ainda não estão completamente elucidados na literatura. Sugere-se que essas ações podem ser atribuídas a componentes específicos destes fungos como as â-glucanas, os triterpenos, as lectinas, a arginina, a glutamina e o ergosterol. Investigadores destacam o papel da â-glucana como principal responsável pelos efeitos hipolipidêmicos apresentados pelos fungos medicinais ${ }^{1,2,16}$.

Em relação aos níveis séricos de TG, averiguouse, no grupo placebo deste estudo, aumento significativo com três meses e após seis meses de acompanhamento. Enquanto no grupo Agaricus sylvaticus houve, no mesmo período, redução de TG, apesar de não-significativa, inferindo novamente que o fungo Agaricus sylvaticus é capaz de modular o metabolismo lipídico por meio de componentes biologicamente ativos.

Estudos experimentais conduzidos em animais de laboratório têm comprovado que a administração de determinadas espécies de fungos medicinais é capaz de promover redução significativa do CT, LDL-c ${ }^{4,5,17-20}$, VLDL-c ${ }^{5,17}, \mathrm{TG}^{16-20}$, fosfolipídio, índice aterogênico e da atividade da enzima 3-hidroxi-3-metilglutarilcoenzima A redutase (HMG-CoA redutase), além do aumento do HDL-c ${ }^{20}$.

Hipóteses sugerem que os fungos medicinais são capazes de reduzir os níveis lipídicos por meio do aumento da excreção fecal e, particularmente, por aumentar o receptor hepático LDL, efeitos estes proporcionados pelas fibras alimentares ${ }^{5}$. As lovastatinas, substâncias que se ligam às estatinas, inibidoras da enzima HMG-CoA redutase, que catalisa a síntese do mevalonato, podem ser detectadas em determinados fungos medicinais e, dessa forma, atuar conjuntamente como responsáveis pelos efeitos observados ${ }^{19,21}$. Além disso, estudos identificaram a presença de eritadenina, agente hipolipidêmico, presente nos fungos medicinais, capaz de reduzir os níveis de colesterol e outros lipídeos por meio da excreção do colesterol ingerido e de sua decomposição metabólica ${ }^{17,19}$, confirmando que diversas substâncias desconhecidas podem estar presentes nos fungos e responder igualmente pelos atributos hipolipidêmicos.

Estudos clínicos são escassos na literatura, porém, Novaes $e t a l$, em um ensaio clínico randomizado, duplo-cego e placebo-controlado, comprovaram que a suplementação com fungo Agaricus sylvaticus em pacientes no pós-operatório de câncer colorretal, foi capaz de reduzir os níveis de CT ( $p=0.01)$ e LDL-c $(p>$ 0.05 ) comparado com o grupo placebo. Ainda neste estudo, os autores observaram que os níveis de HDL-c e VLDL-c não alteraram em ambos os grupos. Resultados similares foram encontrados no nosso estudo, uma vez que os valores de HDL-c e VLDL-c, nos grupos placebo e Agaricus sylvaticus, também não apresentaram alterações estatisticamente significantes durante todo o período de acompanhamento.

Pesquisas científicas têm comprovado que a principal substância que responde pelos atributos funcionais dos fungos medicinais é a â-glucana ${ }^{1-3,16}$. As âglucanas, fibras alimentares solúveis, são capazes de atuar eficazmente na redução do colesterol e de outros lipídeos plasmáticos ${ }^{16,17,22}$. Entretanto, os mecanismos de ação dessas substâncias bioativas ainda não estão totalmente esclarecidos ${ }^{6}$.

Estudos demonstram que a excreção aumentada de ácidos biliares nas fezes ocorre devido à ingestão de fungos medicinais, fontes de fibras, e que a hipótese preliminar a respeito do mecanismo redutor dos níveis de colesterol é o incremento da excreção fecal de ácidos biliares e de colesterol ${ }^{17}$.

Outras hipóteses indicam que as fibras solúveis promovem alterações na absorção intestinal, na secreção de hormônios pancreático e/ou intestinal, no metabolismo das lipoproteínas e dos ácidos biliares e na fermentação dos produtos e seus efeitos na síntese hepática de colesterol, além de aumentar a viscosidade no intestino delgado, resultando na reduzida absorção e/ou reabsorção de ácidos biliares e de colesterol ou triglicerídeos, reduzindo conseqüentemente os níveis lipídicos plasmáticos. Além disso, os ácidos graxos de cadeia curta, particularmente o propionato, um metabólito fermentável das fibras solúveis, podem estar envolvidos na redução das concentrações séricas de colesterol. A atividade da HMG-CoA redutase é também regulada por modificações nas concentrações exógenas de colesterol ${ }^{5,6}$. 
Rev bras Coloproct Julho/Setembro, 2008
Alterações Lipídicas em Pacientes com Câncer Colorretal em Fase Pós-Operatória: Ensaio Clínico Randomizado e Duplo-Cego com Fungos Agaricus sylvaticus Renata Costa Fortes e Cols.
Vol. 28 $\mathbf{N}^{\circ} 3$

\section{CONCLUSÃO}

Os resultados sugerem que, a suplementação dietética com fungos Agaricus sylvaticus é capaz de reduzir o colesterol total, LDL-c e triglicérides, apresentando efeitos benéficos no metabolismo lipídico e, conseqüentemente, no prognóstico desses pacientes.

\begin{abstract}
Introduction: Alterations in the lipidic metabolism are common in patients with cancer. Medicinal fungus may show hypolipidemic activity. Objective: To evaluate the effects of dietary supplementation with Agaricus sylvaticus fungus in lipidic profile of patients with colorectal cancer in post-surgery phase. Methods: Randomized, double-blind, placebo-controlled clinical trial carried out at the Federal District Base Hospital, for six months. Samples of 56 enrolled patients, stadiums phase I, II and III, divided in two groups: placebo group and group supplemented with Agaricus sylvaticus $(30 \mathrm{mg} / \mathrm{kg} / \mathrm{day})$. Results were analyzed with Microsoft Excel 2003 and SPSS 14.0 programs, with $p=0.05$. Results: The Agaricus sylvaticus group had initial total cholesterol levels of $207.36 \pm 52.67 \mathrm{mg} / \mathrm{dL}$, low-density lipoprotein of $120.79 \pm 44.02 \mathrm{mg} / \mathrm{dL}$ and triglycerides of $181.64 \pm 187.52 \mathrm{mg} / \mathrm{dL}$. After six months of supplementation it was observed a reduction $191.11 \pm 39.72 \mathrm{mg} / \mathrm{dL}(p=0.01), 103.08 \pm 39.20 \mathrm{mg} / \mathrm{dL}(p=0.0001)$ and $168.04 \pm 146.91 \mathrm{mg} / \mathrm{dL}(p=0.18)$, respectively. In the placebo group it was observed not-significant increase of total cholesterol levels $(p=0.08)$ and significant increase of low-density lipoprotein $(p=0.01)$ and triglycerides $(p=0.0001)$. No significant statistically alterations were observed in the levels of high-density lipoprotein and very-low-density lipoprotein in both groups. Conclusion: Results suggest that the dietary supplementation with Agaricus sylvaticus may significantly improve the lipidic profile of colorectal cancer patients in post-surgery phase.
\end{abstract}

Keywords: Agaricus sylvaticus, medicinal fungus, metabolic alterations, lipidic metabolism, colorectal cancer.

\section{REFERÊNCIAS}

1. Fortes RC, Taveira VC, Novaes MRCG. The immunomodulator role of $\hat{a}-\mathrm{D}$-glucans as co-adjuvant for cancer therapy. Rev Bras Nutr Clin 2006; 21(2): 163-8.

2. Fortes RC, Novaes MRCG. Efeitos da suplementação dietética com cogumelos Agaricales e outros fungos medicinais na terapia contra o câncer. Rev Bras Cancerol 2006; 52(4): 363-71.

3. Novaes MRCG, Fortes RC. Efeitos antitumorais de cogumelos comestíveis da família agaricaceae. Rev Nutr Bras 2005; 4(4): 207-217.

4. Berger A, Jones PJH, Abumweis SS. Plant sterols: factors affecting their efficacy and safety as functional. Lipids in Health and Disease 2004; 3:5.

5. Fukushima M, Ohashi T, Fujiwara Y, Sonoyama K, Nakano M. Cholesterol-lowering effects of maitake (Grifola frondosa) fiber, shiitake (Lentinus edodes) fiber, and enokitake (Flammulina velutipes) fiber in rats. Exp Biol Med 2001; 226(8): 758-65.

6. Fukushima M, Nakano M, Morii Y, Ohashi T, Fujiwara Y, Sonoyama K. Hepatic LDL receptor mRNA in rats is increased by dietary mushroom (Agaricus bisporus) fiber and sugar beet fiber. J. Nutr 2000; 130: 2151-56.

7. Novaes MRCG, Fortes RC, editors. Alterations on the metabolism of lipids in post-surgery patients with colorectal cancer supplemented with fungus Agaricus sylvaticus. Proceedings of the $27^{\text {th }}$ International Congress of ESPEN and Clinical Nutrition; 2005 Aug 27-30; Brussels, Belgium: Elsevier 2005.
8. Recomendações da Sociedade Brasileira de Patologia Clínica / Medicina Laboratorial para Coleta de Sangue Venoso. $1^{\mathrm{a}}$ edição. São Paulo. 2005: 01-76.

9. Pinho MSL; Ferreira LC; Brigo MJK; Pereira Filho A; Wengerkievicz A; Ponath A; Salmoria L. Incidência do câncer colorretal na regional de saúde de Joinville (SC). Rev bras Coloproct, 2003; 23(2): 73-76.

10. Saad-Hossne R, Prado RG, Bakonyi Neto A, Lopes PS, Nascimento SM, Santos CRV, et al. Estudo retrospectivo de pacientes portadores de câncer colorretal atendidos na Faculdade de Medicina de Botucatu no período de 2000-2003. Rev bras Coloproct, 2005; 25(1): 31-37.

11. Waitzberg DL, Mazza RPJ, Alves CC. Conseqüências nutricionais do tratamento cirúrgico do trato gastrintestinal. In: Waitzberg DL: Dieta, Nutrição e Câncer. São Paulo: Atheneu, 2004. p. 407-24.

12. Brasil. Ministério da Saúde. Secretaria de Atenção à Saúde. Instituto Nacional do Câncer. Coordenação de Prevenção e Vigilância. Estimativa 2005: Incidência de câncer no Brasil. Rio de Janeiro: INCA, 2004. 94p: il.

13. Faria FA, Campos CS, Fortes RC. Terapia nutricional enteral em pacientes oncológicos: uma revisão da literatura. Com Ciências Saúde 2008; 19(1): 61-70.

14. Montagnini AL, Perini MV. Alterações metabólicas do câncer. In.: Ikemori EHA, Oliveira T, editores. Nutrição em oncologia. São Paulo: Editora Marina e Tecmedd; 2003. p. 35-44.

15. Rocha EEM. Ciclos metabólicos fúteis em câncer. In: Waitzberg DL. Dieta, nutrição e câncer. São Paulo: Atheneu, 2004. p. 297-320. 
16. Taveira VC, Novaes MRCG. Consumo de cogumelos na nutrição humana: uma revisão da literatura. Com. Ciências Saúde 2007; 18(4): 315-22.

17. Cheung PCK. Plasma and hepatic cholesterol levels and fecal neutral sterol excretion are altered in hamsters fed straw mushroom diets. The Journal of Nutrition 1998; 128 (9): 151216.

18. Toews VD. Power your immunity with mushroom. Better Nutrition 1999; 61(4): 58-60.

19. Wasser SP, Weis AL. Medicinal properties of substances occurring in higher basidiomycetes mushrooms: current perspectives. Int J Med Mushrooms 1999; 1:31-62.

20. Yang BK, Park JB, Song CH. Hypolipidemic effect of an exobiopolymer produced from a submerged mycelial culture of Hericium erinaceus. Biosci Biotechnol Biochem 2003; 67(6): 1292-98.
21. Lindequist $\mathrm{U}$, Niedermeyer THJ, Jülich W-D. The pharmacological potential of mushrooms. eCAM 2005; 2(3): 285-99.

22. Biörklund M, Rees AV, Mensink RP, Önning G. Changes in serum lipids and postprandial glucose and insulin concentrations after consumption of beverages with â-glucans from oats or barley: a randomized dose-controlled trial. European Journal of Clinical Nutrition 2005; 59(11): 127281.

Endereço para correspondência: RENATA COSTA FORTES

QI 14. CJ J. CS 26. Guará 1 / DF

CEP: $71.015-100$

Telefone: (0xx61) 9979-9463

E-mail: renatacfortes@yahoo.com.br 